\title{
Structure and Bonding of Iron-Acceptor Pairs in Silicon
}

\author{
S. Zhao, \\ Texas Instruments Inc., Dallas, TX, 75243 \\ J. F. Justo, L. V. C. Assali, \\ Instituto de Física, Universidade de São Paulo, \\ CP 66318, CEP 05315-970, São Paulo, SP, Brazil \\ and L. C. Kimerling \\ Massachusetts Institute of Technology, Cambridge, MA, 02139
}

Received on 23 April, 2001

\begin{abstract}
Iron-acceptor pairs (Fe- $A, A=\mathrm{B}, \mathrm{Al}, \mathrm{Ga}$, and $\mathrm{In}$ ) in silicon were investigated using an ionicbased model, which incorporates the valence electron cloud polarization and the lattice relaxation. Our results are generaly in good agreement with the experimental trends among the Fe- $A$ pairs, describing the increase in the pair donor energy level with increasing $A$ principal quantum number and decreasing pair separation distance, and the pair configurational symmetries.
\end{abstract}

Iron pairs with acceptor impurities in silicon [1], forming electrically active centers. The properties of these pairs, such as the configurational structure and the positions of the energy levels in the band gap, have been investigated by electron paramagnetic resonance (EPR) and deep level transient spectroscopy (DLTS) [2] over the last thirty years. These pairs have been identified as consisting of a substitutional acceptor $\left(A_{s}\right)$ with an iron $\left(\mathrm{Fe}_{i}\right)$ at either the nearest neighbor $(\mathrm{T} 1,\langle 111\rangle$ symmetry) or the next nearest neighbor (T2, $\langle 100\rangle$ symmetry) tetrahedral interstitial site. According to an ionic model, used to explain the experimental data, the $\mathrm{Fe}_{i} A_{s}$ pairs are formed by a positively charged iron $\left(\mathrm{Fe}_{i}^{+}\right.$ or $\left.\mathrm{Fe}_{i}^{++}\right)$and a negatively charged acceptor $\left(A_{s}^{-}\right)$.

The ionic model fails in describing several trends among the $\mathrm{Fe}_{i} A_{s}$ pairs, such as the pair stability and the related positions of the acceptor and donor electronic levels [3]. Adding an elastic energy term to the point charge interaction, Kimerling et al. [4] explained the pair structures observed by the experiments. They noticed that large acceptor impurities provide strong repulsion enough to compete with the Coulomb attraction so that the $\mathrm{Fe}_{i}$ stays at a T2 site (and not at a $\mathrm{T} 1$ site) next to the acceptor. Our model incorporates a new interaction in the point charge Coulomb interaction, a short range attractive component to simulate the valence electronic cloud polarization [5]. The repulsive interaction between $\mathrm{Fe}_{i}$ and $A_{s}$ or $\mathrm{Si}$ is approximated by a softened Lennard-Jones type potential, and the silicon crystal is treated as a dielectric medium. The results are compared to the experimental data on configurational symmetries and deep level positions. Our model captured several trends on the pairs observed by experiments [2], showing an increase in the pair donor energy level with increasing principal quantum number of $A_{s}$ and decreasing pair separation distance, opposite to results obtained by Kimerling et al. [4].

When the separation between $\mathrm{Fe}_{i}$ and $A_{s}$ is comparable to their ionic radii, the electronic cloud of one ion is strongly perturbed by the field of the other, causing an induced polarization on that electronic cloud. Our polarization model (PM) includes a short range attractive component to the Coulomb interaction to describe the electronic cloud polarization. As an estimation to the induced electronic cloud polarization effect, we take the valence electronic cloud as a charged spherical surface at certain radius around the radial peak position of the valence electronic charge density. Then, similar to the interaction between two conducting spheres, the electrostatic interaction can be written as $\left(r \equiv r_{\mathrm{Fe}-\mathrm{A}}\right)$

$$
V_{\text {att }}(r)=\frac{1}{4 \pi \epsilon_{r} \epsilon_{0}}\left[\frac{q_{1} q_{2}}{r}-\frac{1}{2}\left(\frac{a_{2}^{3} q_{1}^{2}}{r^{4}}+\frac{a_{1}^{3} q_{2}^{2}}{r^{4}}\right)\right],
$$

where $a_{1}$ and $a_{2}$ are the valence electronic cloud spherical surface radii, $q_{1}$ and $q_{2}$ are the net ionic charges.

The calculations were performed using $279 \mathrm{Si}$ atoms, one $A_{s}$, and one $\mathrm{Fe}_{i}$. The distance $r_{\mathrm{Fe}-\mathrm{A}}$ is changed along $\langle 111\rangle$ direction (passing through $\mathrm{T} 1$ and $\mathrm{T} 4$ sites) and $\langle 100\rangle$ direction (passing through T2 site) to find the potential curves for $\mathrm{Fe}_{i}^{0}, \mathrm{Fe}_{i}^{+}$, and $\mathrm{Fe}_{i}^{++}$. The minimum of each curve $V_{\text {min }}\left(\mathrm{Fe}_{i}^{0}\right), V_{\min }\left(\mathrm{Fe}_{i}^{+}\right)$, and $V_{\text {min }}\left(\mathrm{Fe}_{i}^{++}\right)$is the ground state for $\mathrm{Fe}_{i}^{0}, \mathrm{Fe}_{i}^{+}$, and $\mathrm{Fe}_{i}^{++}$, respectively. The model predicts the $\mathrm{Fe}_{i} A_{s}$ pair configurational symmetries by determining the energetically favorable sites for $\mathrm{Fe}_{i}$. 


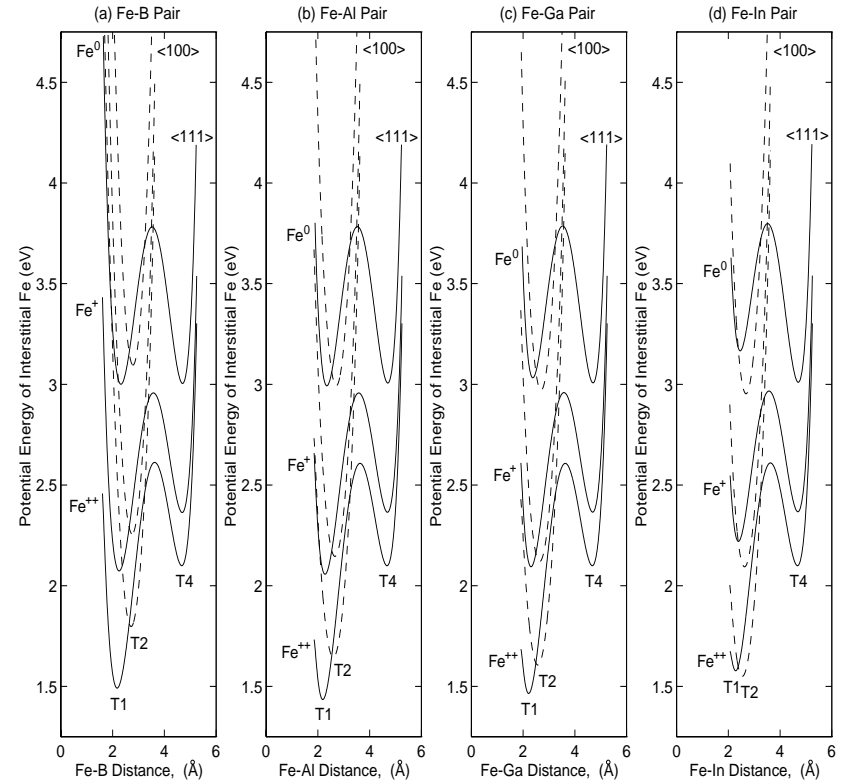

Figure 1. The role of $\mathrm{Fe}_{i}$ charge state on the $\mathrm{Fe}_{i} A_{s}$ pair symmetry and stability. The potential curves along $\langle 111\rangle$ and $\langle 100\rangle$ directions are represented by solid and dashed lines, respectively.

Fig. 1 shows the potential curves along $\langle 111\rangle$ and $\langle 100\rangle$ directions for $\mathrm{Fe}_{i}^{0}, \mathrm{Fe}_{i}^{+}$and $\mathrm{Fe}_{i}^{++}$. According to Figs. $1 \mathrm{a}$ and $1 \mathrm{~d}$, stable $\mathrm{Fe}_{i} \mathrm{~B}_{s}$ and metastable $\mathrm{Fe}_{i} \mathrm{In}_{s}$ pairs have $\langle 111\rangle$-trigonal symmetry, while stable $\mathrm{Fe}_{i} \mathrm{In}_{s}$ and metastable $\mathrm{Fe}_{i} \mathrm{~B}_{s}$ pairs have $\langle 100\rangle$-orthorhombic symmetry. The $\langle 111\rangle$-trigonal symmetry can be assigned to stable $\mathrm{Fe}_{i}^{+} \mathrm{Al}_{s}^{-}, \mathrm{Fe}_{i}^{++} \mathrm{Al}_{s}^{-}, \mathrm{Fe}_{i}^{+} \mathrm{Ga}_{s}^{-}$, and $\mathrm{Fe}_{i}^{++} \mathrm{Ga}_{s}^{-}$pairs, while metastable $\mathrm{Fe}_{i}^{+} \mathrm{Al}_{s}^{-}, \mathrm{Fe}_{i}^{++} \mathrm{Al}_{s}^{-}$, $\mathrm{Fe}_{i}^{+} \mathrm{Ga}_{s}^{-}$, and $\mathrm{Fe}_{i}^{++} \mathrm{Ga}_{s}^{-}$pairs show $\langle 100\rangle$ symmetry (Figs. $1 \mathrm{~b}$ and 1c). In addition, Figs. $1 \mathrm{~b}$ and $1 \mathrm{c}$ predict that the stable $\mathrm{Fe}_{i}^{0} \mathrm{Ga}_{s}^{-}$pair exhibits $\langle 100\rangle$ symmetry, while the stable $\mathrm{Fe}_{i}^{0} \mathrm{Al}_{s}^{-}$pair has equal probability to show either $\langle 111\rangle$-trigonal or $\langle 100\rangle$-orthorhombic structures. The assignments for stable and metastable structures are in good agreement with the EPR and DLTS observations [2]. The stable configuration switches from $\langle 111\rangle$-trigonal to $\langle 100\rangle$-orthorhombic for $\mathrm{Fe}_{i} \mathrm{~A}_{s}$ pairs going from $\mathrm{B}_{s}$ to $\mathrm{In}_{s}$, and is related to an increase in repulsion between $\mathrm{A}_{s}$ and $\mathrm{Fe}_{i}$.

The minimum of each curve can be used to compute the pair acceptor and donor levels. For $\mathrm{Fe}_{i}$ at near neighbor $\mathrm{T}$ sites relative to $A_{s}$, the donor level $\left(\mathrm{Fe}_{i}^{+/++} A_{s}^{-}\right)^{0 /+}$ is given by

$$
E_{T}(0 /+)=E_{v}+V_{\min }\left(\mathrm{Fe}_{i}^{+}\right)-V_{\min }\left(\mathrm{Fe}_{i}^{++}\right)+E_{T}^{0},
$$

where $E_{v}$ is the top of the valence band and $E_{T}^{0}$ is the minimum of the $\mathrm{Fe}_{i}^{++}$potential curve at near neighbor sites of $A_{s}^{-}$. The pair acceptor level $\left(\mathrm{Fe}_{i}^{0 /+} A_{s}^{-}\right)^{-/ 0}$ is obtained by adding the difference $V_{\min }\left(\mathrm{Fe}_{i}^{0}\right)-V_{\min }\left(\mathrm{Fe}_{i}^{+}\right)$to the donor level. For isolated $\mathrm{Fe}_{i}$, at remote sites relative to $A_{s}, V_{\min }\left(\mathrm{Fe}_{i}^{0}\right)-V_{\min }\left(\mathrm{Fe}_{i}^{+}\right)=0.38 \mathrm{eV}$, which is consistent with experimental data that only one donor level
$\mathrm{Fe}_{i}^{0 /+}$ at $E_{v}+0.38 \mathrm{eV}$ exists in the band gap. In our model, the difference between $V_{\min }\left(\mathrm{Fe}_{i}^{0}\right)$ and $V_{\min }\left(\mathrm{Fe}_{i}^{+}\right)$ for isolated $\mathrm{Fe}_{i}$ arises from the elastic energy caused by electron shell overlap between atoms. Fig. 2 shows the donor $(0 /+)$ and acceptor $(-/ 0)$ levels for the transitions as computed by our polarization model for $\mathrm{Fe}_{i}$ sitting at T1 (Fig. 2a) or T2 (Fig. 2b) sites. Our results for the pair donor and acceptor levels agree very well with the known experimental data for the $(0 /+)$ and $(-/ 0)$ transitions.

The PM predicts the correct magnitudes and trends of the donor level for the T1 site $\left(E_{T}(1)\right)$ and the T2 site $\left(E_{T}(2)\right)$ pairs with increasing $A_{s}$ size and decreasing $r_{\mathrm{Fe}-\mathrm{A}}$ as observed in experiments, while the point charge model would give an opposite trend. According to our model, repulsion and polarization from $A_{s}$ should give a maximum contribution at the $\mathrm{T} 1$ site and yield the greatest variation in $\mathrm{Fe}_{i} A_{s}$ pair energy levels. This is consistent with the experimental data that $E_{T}(1)$ displays the greatest sensitivity to $A_{s}$ identity and $E_{T}(2)$ shows relative uniformity. The PM suggests that the pair acceptor level $\left(\mathrm{Fe}_{i}^{0 /+} A_{s}^{-}\right)^{-/ 0}$ should become shallower in the band gap as $A_{s}$ goes from $\mathrm{B}_{s}$ to $\mathrm{In}_{s}$, while recent experimental data [6] suggest that this level is almost constant for $A_{s}$.

(a)
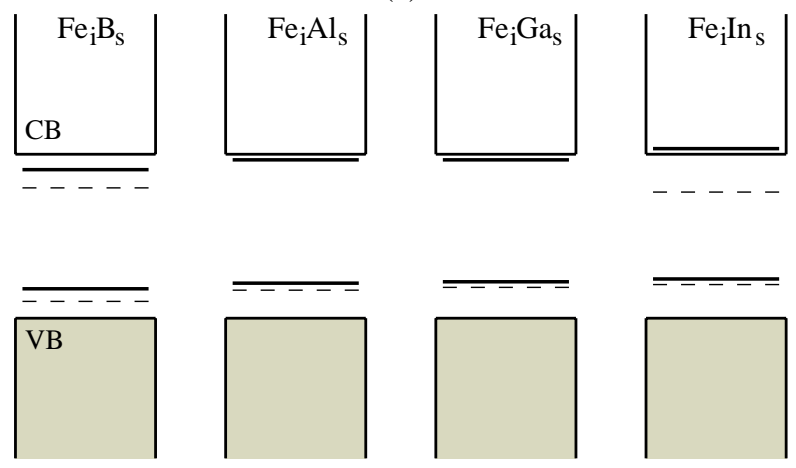

(b)
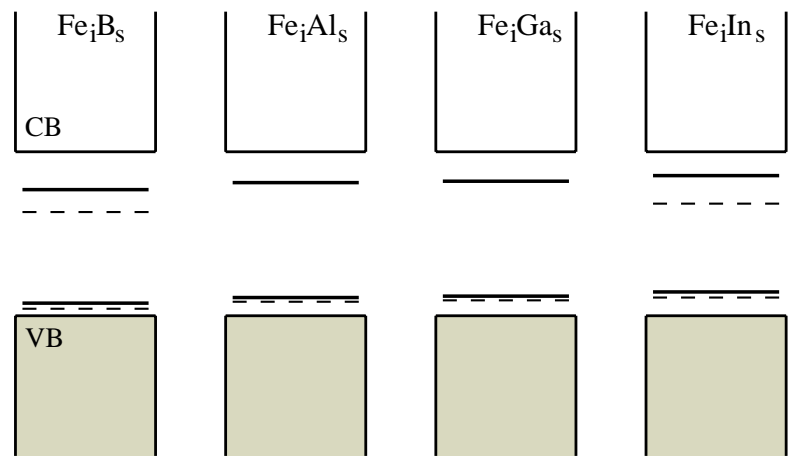

Figure 2. Representation of donor and acceptor levels, corresponding to $(0 /+)$ and $(-/ 0)$ transitions, for $\mathrm{Fe}_{i} A_{s}$. The figure shows the levels for the $\mathrm{Fe}_{i}$ sitting at a $\mathrm{T} 1$ site (a) and at a T2 site (b). Dashed lines represent the available experimental results for the respective transitions [2]. VB and $\mathrm{CB}$ represent the valence and conduction bands. 
Table I. Pair binding energies $E_{b}$ (in $\mathrm{eV}$ ), computed using the polarization model, for stable $\mathrm{Fe}_{i} A_{s}$ in $\mathrm{Si}$, compared to experimental data.

\begin{tabular}{lcc}
\hline $\mathrm{Fe}_{i} A_{s}$ & $E_{b}^{e x p}$ & $E_{b}$ \\
\hline $\mathrm{Fe}_{i}^{+} \mathrm{B}_{s}^{-}$ & $0.65[3], 0.58[7]$ & 0.56 \\
$\mathrm{Fe}_{i}^{+} \mathrm{Al}_{s}^{-}$ & $0.52[8]$ & 0.58 \\
$\mathrm{Fe}_{i}^{+} \mathrm{Ga}_{s}^{-}$ & & 0.53 \\
$\mathrm{Fe}_{i}^{+} \mathrm{In}_{s}^{-}$ & & 0.55 \\
\hline $\mathrm{Fe}_{i}^{++} \mathrm{B}_{s}^{-}$ & & 1.13 \\
$\mathrm{Fe}_{i}^{++} \mathrm{Al}_{s}^{-}$ & $1.04[8]$ & 1.19 \\
$\mathrm{Fe}_{i}^{++} \mathrm{Ga}_{s}^{-}$ & & 1.16 \\
$\mathrm{Fe}_{i}^{++} \mathrm{In}_{s}^{-}$ & & 1.09 \\
\hline
\end{tabular}

The pair binding energies are shown in Table I. The model predicts a trend of monotonic decrease with increasing $A_{s}$ size for both $\mathrm{Fe}_{i}^{+}$and $\mathrm{Fe}_{i}^{++}$. The increase in binding energy due to valence electronic cloud polarization competes with the repulsive interaction so that the model gives nearly constant binding energies for both $\mathrm{Fe}_{i}^{+}$and $\mathrm{Fe}_{i}^{++}$, consistent with experimental data $[3,7,8]$. Lattice relaxation clearly plays an important role in determining the pair binding energy.

The relative population of $\mathrm{Fe}_{i} A_{s}$ pairs in a certain charge state at T1 and T2 sites $\left(R_{12}\right)$ is calculated, based on a Boltzmann distribution at thermal equilibrium:

$$
R_{12}=\frac{N(1)}{N(2)}=\frac{Z_{1}}{Z_{2}} \exp \left(\frac{\Delta E_{12}}{k T}\right),
$$

where $\Delta E_{12}=E_{\min }(T 2)-E_{\min }(T 1)$ is the energy difference for $\mathrm{Fe}_{i}$ at the $\mathrm{T} 1$ and $\mathrm{T} 2$ sites, and $Z$ is the site degeneracy. The relative site populations for the pairs are compared with results from metastability experiments in Table II. Our model provides a good description of the energy differences between the $\mathrm{T} 1$ and T2 sites $\left(\Delta E_{12}\right)$ for all the pairs. The calculated $N(1) / N(2)$ ratios at $T=200 \mathrm{~K}$, around the temperature of the observed structural transformation $[8,9]$, also agree very well with the experimental data.

Table II. Calculated relative site populations $\left(R_{12}\right)$ at $\mathrm{T}$ $=200 \mathrm{~K}$ and energy difference $\left(\Delta E_{12}\right)$, between centers with $\mathrm{Fe}_{i}$ at $\mathrm{T} 1$ and $\mathrm{T} 2$ sites for the pairs. Experimental results are in parenthesis.

\begin{tabular}{lcc}
\hline & $R_{12}$ & $\Delta E_{12}(\mathrm{eV})$ \\
\hline $\mathrm{Fe}_{i}^{+} \mathrm{B}_{s}^{-}$ & $4.1 \times 10^{4}(\infty[4])$ & $0.19(>0.1[4])$ \\
$\mathrm{Fe}_{i}^{+} \mathrm{Al}_{s}^{-}$ & $1.2 \times 10^{2}(2.2[4])$ & $0.09(0.07[8])$ \\
$\mathrm{Fe}_{i}^{+} \mathrm{Ga}_{s}^{-}$ & $3.8(7.0[4])$ & $0.03(0.03[10])$ \\
$\mathrm{Fe}_{i}^{+} \mathrm{In}_{s}^{-}$ & $3.5 \times 10^{-4}(0.0[4])$ & $-0.13(-0.13[9])$ \\
\hline $\mathrm{Fe}_{i}^{++} \mathrm{B}_{s}^{-}$ & $4.3 \times 10^{7}(\infty[4])$ & $0.31(>0.1[4])$ \\
$\mathrm{Fe}_{i}^{++} \mathrm{Al}_{s}^{-}$ & $1.3 \times 10^{5}(\infty[4])$ & $0.21(0.14[8])$ \\
$\mathrm{Fe}_{i}^{++} \mathrm{Ga}_{s}^{-}$ & $4.0 \times 10^{3}(\infty[4])$ & $0.15(0.13[9])$ \\
$\mathrm{Fe}_{i}^{++} \mathrm{In}_{s}^{-}$ & $0.12(0.42[4])$ & $-0.03(-0.01[9])$ \\
\hline
\end{tabular}

In summary, our model captures several effects within the ionic model framework: (i) trends among the $\mathrm{Fe}_{i} A_{s}$ pairs revealing a deepening of the donor level in the band gap with increasing principal quantum number of $A_{s}$ and decreasing pair separation distance $r_{\mathrm{Fe}-\mathrm{A}}$; and (ii) configurational symmetries and the bistability of the pairs. However, the deviations at $E_{T}(1)$ between measured and calculated data suggest that other interactions are still missing. The deviations could come from the limitations of the ionic models at near neighbor $\mathrm{T}$ sites. The bulk, treated as dielectric medium, can still be valid for the space between a $\mathrm{T} 4$ site and the $A_{s}$, and between a T2 site and the $A_{s}$. However, at the $\mathrm{T} 1$ site, the screening would hardly be effectively described by the bulk dielectric constant, since one of the $\mathrm{Fe}_{i}$ first neighbors is the $A_{s}$. Covalency involving the $\mathrm{Fe}_{i}, A_{s}$, and surrounding $\mathrm{Si}$ atoms may also play an important role, as pointed out by first-principles calculations $[11,12,13]$. Although a complete understanding on the properties of the pairs should be established with more detailed experiments and calculations, our model is important in identifying the contribution of each interaction for the pair formation.

\section{Acknowledgments}

This work is supported by the NREL under contract No. XD-2-11004-4. JFJ and LVCA thank the Brazilian agencies FAPESP and CNPq for financial support.

\section{References}

[1] G. W. Ludwig and H. H. Woodbury, Solid State Phys. 13, 223 (1962).

[2] A. A. Istratov, H. Hieslmair, and E. R. Weber, Appl. Phys. A 69, 13 (1999).

[3] L. C. Kimerling and J. L. Benton, Physica 116B, 297 (1983).

[4] L. C. Kimerling, M. T. Asom, J. L. Benton, P. J. Drevinsky, and C. E. Caefer, Mater. Sci. Forum 3841, 141 (1989).

[5] S. Zhao, L. V. C. Assali, J. F. Justo, G. H. Gilmer, and L. C. Kimerling, J. Appl. Phys. 90, 2744 (2001).

[6] P. Tidlund, M. Kleverman, and H. G. Grimmeiss, Semicond. Sci. Technol. 11, 748 (1996).

[7] W. Wijaranakula, J. Electrochem. Soc. 140, 275 (1993).

[8] A. Chantre and D. Bois, Phys. Rev. B 31, 7979 (1985).

[9] A. Chantre and L. C. Kimerling, Mater. Sci. Forum 10-12, 387 (1986).

[10] H. Takahashi, M. Suezawa, and K. Sumino, Mater. Sci. Forum 83-87, 155 (1992).

[11] L. V. C. Assali and J. R. Leite, Mater. Sci. Forum 1012, 55 (1986); 38-41, 409 (1989); 83-7, 143 (1992).

[12] L. V. C. Assali and J. F. Justo, Phys. Rev. B 58, 3870 (1998).

[13] J. F. Justo and L. V. C. Assali, Int. J. Mod. Phys. B 13, 2387 (1999). 